\title{
The Long Non-Coding RNA XIST Controls Non-Small Cell Lung Cancer Proliferation and Invasion by Modulating miR-186-5p
}

\author{
Haoyou Wang Qiming Shen Xin Zhang Chunlu Yang Su Cui Yanbin Sun \\ Liming Wang Xiaoxi Fan Shun Xu \\ Department of Thoracic Surgery, The First Hospital of China Medical University, 155 North Nanjing \\ Street, Heping District, Shenyang City, Liaoning Province, China
}

\author{
Key Words \\ IncRNA XIST • NSCLC • miR-186-5p • Invasion
}

\begin{abstract}
Background/Aims: Long non-coding RNAs (IncRNAs) are key players in the development and progression of human cancers. The IncRNA XIST (X-inactive specific transcript) has been shown to be upregulated in human non-small cell lung cancer (NSCLC); however, its role and molecular mechanisms in NSCLC cell progression remain unclear. Methods: qRT-PCR was conducted to assess the expression of XIST and miR-186. Cell proliferation was detected using MTT assay. Cell invasion and migration were evaluated using transwell assay. Cell cycle distribution and apoptosis rates were analyzed by flow cytometry. Luciferase reporter assay was used to identify the direct regulation of XIST and miR-186. A RNA immunoprecipitation was used to analyze whether XIST was associated with the RNA-induced silencing complex (RISC). Results: We confirmed that XIST was upregulated in NSCLC cell lines and tissues. Functionally, XIST knockdown inhibited cancer cell proliferation and invasion, and induced apoptosis in vitro, and suppressed subcutaneous tumor growth in vivo. Mechanistic investigations revealed a reciprocal repressive interaction between XIST and miR-186-5p. Furthermore, we showed that miR-186-5p has a binding site for XIST. Our data also indicated that XIST and miR-186$5 p$ are likely in the same RNA induced silencing complex. Conclusion: Together, our data revealed that XIST knockdown confers suppressive function in NSCLC and XIST may be a novel therapeutic marker in this disease.

\section{Introduction}

Non-small cell lung cancer (NSCLC) accounts for approximately $80 \%$ of all lung cancers, which are the primary cause of cancer-related deaths worldwide [1]. Despite advances in the early diagnosis and treatment of lung cancer, the prognosis of lung cancer patients remain poor, with the five-year overall survival rates currently at 18\% [2]. Hence, an improved and 
detailed understanding of the molecular mechanisms underlying NSCLC progression is desperately needed. Recently, increasing evidence has shown that long non-coding RNAs (lncRNAs) provide new insights into the treatment of NSCLC pathogenesis.

Protein-coding genes account for only $2 \%$ of the human genome, whereas the vast majority of transcripts are non-coding RNAs (ncRNAs), including microRNA (miRNAs) and lncRNAs [3]. A number of microRNAs were found to act as oncogenes or tumor suppressor genes by previous reports [4], whereas the mechanism of action for long ncRNAs (lncRNAs) remains largely unknown. LncRNAs are a class of non-coding RNA greater than 200 nucleotides with no protein-coding potential [5]. LncRNAs play critical roles in numerous biological processes, such as epigenetic regulation, nuclear import, cell cycle control, imprinting, differentiation, alternative splicing, RNA decay and transcription [6, 7]. Recent studies have revealed that the expression of IncRNAs is frequently dysregulated in cancers including NSCLC, and is usually correlated with cancer progression, metastasis and poor prognosis $[8,9]$. For example, Wan et al. found that the lncRNA plasmacytoma variant translocation 1 (PVT1) promoted NSCLC proliferation by epigenetically regulating large tumor suppressor kinase 2 (LATS2) expression [10]. LncRNA- urothelial carcinoma-associated 1 (UCA1) exerts oncogenic functions in NSCLC by targeting miR-193a-3p [11]. A complete understanding of the roles of IncRNAs in NSCLC would greatly advance the development of novel therapeutic strategies.

The lncRNA XIST (X-inactive specific transcript), a product of the XIST gene, is the master regulator of $\mathrm{X}$ inactivation in mammals, and XIST is exclusively transcribed from the inactive X chromosome [12]. XIST is highly expressed in some carcinomas including breast cancer [13], glioblastoma [14] and ovarian cancer [15], suggesting that XIST may serve as a biomarker for the diagnosis of these cancers. A recent study showed that XIST is upregulated and is essential for the long term survival of NSCLC [16]; however, its molecular role in NSCLC has not been clarified.

In this study, we first confirmed that XIST expression was increased in NSCLC cell lines and tissues. Next, we showed that XIST knockdown inhibited NSCLC proliferation and invasion in vitro and suppressed tumor growth in vivo. Finally, miR-186-5p was found to block the oncogenic functions of XIST in NSCLC cells. Therefore, our study identifies XIST as a novel therapeutic target for NSCLC treatment.

\section{Materials and Methods}

\section{Tissue specimens}

We obtained 30 paired NSCLC and adjacent non-cancerous lung tissues from patients who had undergone surgeries at The First Hospital of China Medical University. All patients had not received chemotherapy or radiotherapy before surgery. All collected tissue samples were immediately snap-frozen in liquid nitrogen and stored at $-80^{\circ} \mathrm{C}$ until total RNA was extracted. This study was approved by The First Hospital of China Medical University, and it was performed in compliance with the Declaration of Helsinki Principles. Written informed consent was obtained from all patients.

\section{Cell culture and transfection}

The NSCLC cell lines (A549, H1299, SK-MES-1 and Calu-3) and the immortalized human bronchial epithelial cell line (BEAS-2B) were purchased from the Institute of Biochemistry and Cell Biology of the Chinese Academy of Sciences (Shanghai, China). The human NSCLC cell lines were cultured in Dulbecco's Modified Eagle's medium (DMEM) (Invitrogen, Carlsbad, CA, USA) medium or RPMI 1640 medium (Invitrogen). Transfection was performed using Lipofectamine 2000 (Invitrogen) according to the manufacturer's protocol.

Quantitative real-time PCR

Total RNA from tissues and cells was extracted using TRIzol reagent (Invitrogen) according to the manufacturer's instructions. First strand cDNA was generated using the Reverse Transcription System Kit 


\section{Cellular Physiology Cell Physiol Biochem 2017;41:2221-2229 \begin{tabular}{l|l} 
and Biochemistry Published 10.1159/000475637 & $\begin{array}{l}\text { C } 2017 \text { The Author(s). Published by S. Karger AG, Basel } \\
\text { www.karger.com/cpb }\end{array}$
\end{tabular} \\ Wang et al.: LncRNA XIST Controls NSCLC Cell Proliferation and Invasion}

(Takara, Dalian, China). Quantitative real-time PCR (qRT-PCR) analyses utilized SYBR Green I (Takara) and were performed in triplicate. Glyceraldehyde-3-phosphate dehydrogenase (GAPDH) and U6 snRNA were used as endogenous controls. The relative fold change in expression was calculated by the $2^{-\Delta \Delta C t}$ method.

\section{Western blotting}

Total proteins were extracted from cells using RIPA buffer and quantified using a bicinchoninic acid (BCA) protein quantification kit (Beyotime Institute of Biotechnology, Jiangsu, China). Protein was separated using 10\% sodium dodecyl sulfatepolyacrylamide gel electrophoresis (SDS-PAGE) and electrophoretically transferred to polyvinylidene difluoride (PVDF) membrane (Millipore, Billerica, MA, USA). Membranes were incubated with primary antibodies and GAPDH. Immunoreactive bands were visualized using the Pierce ECL Western Blotting Substrate (Santa Cruz Biotechnology).

\section{Plasmid constructs and cell transfection}

Specific siRNA oligonucleotides against XIST (siXIST) and negative control (siNC) were purchased from GenePharma (Shanghai, China). Lentivirus expressing short-hairpin RNA directed against human IncRNA XIST were also purchased from GenePharma. The complementary DNA encoding XIST was PCR-amplified and inserted into pcDNA3.1 (Invitrogen). miR-186-5p mimics and negative control were purchased from Life Technologies (Ambion, Grand Island, NY, USA). The transfection of cells was carried out using Lipofectamine 2000.

Cell viability, cell cycle, cell invasion and apoptosis assay

The 3-(4, 5-dimethylthiazole-2-yl)-2, 5-biphenyl tetrazolium bromide (MTT) assay was performed to test cell viability. Briefly, cells were seeded into 96-well plates at a density of $5 \times 10^{3}$ cells/well. The spectrophotometric absorbance was measured for each sample at $570 \mathrm{~nm}$ at the indicated time points. Cell migration was evaluated by the wound healing assay, cells were seeded in 24-well plates, and the cell monolayer was scratched with the tip of a sterile $200 \mu \mathrm{L}$ pipette tip. The healing process was observed for $24 \mathrm{~h}$ to evaluate the migratory ability of tested cells. The invasive potential was determined using transwell chambers ( $8 \mu \mathrm{m}$ pore; BD Biosciences). Cells were seeded in the top chamber of the inserts, and the cells that invaded to the lower compartment were fixed with methanol and stained with $0.1 \%$ crystal violet. The number of invading cells was calculated by counting five random fields under a microscope. Cell cycle and apoptosis analyses were performed using propidium iodide (Keygen, Nanjing, China) and phycoerythrin (PE) Annexin apoptosis detection kit (BD Pharmingen, San Jose, CA, USA). The cell cycle distribution and apoptosis rates were analyzed by flow cytometry (Epics Altra, Beckman Coulter, USA).

\section{Luciferase reporter assay}

To construct dual luciferase reporter plasmids, the theoretical binding sequence of miR-186-5p in XIST and its mutated sequence were separately cloned into pmirGLO Dual-luciferase vectors (GenePharma). HEK-293T cells were co-transfected with wild-type pmirGLO-XIST (XIST-Wt) or the mutated XIST (XISTMut) reporter plasmid and miR-186-5p mimics or negative control using Lipofectamine 2000 (Invitrogen). After 48h, luciferase activity was detected using the dual-luciferase reporter kit (Promega, Madison, WI, USA). The relative firefly luciferase activity was calculated by normalizing to renilla luciferase activity.

\section{RNA immunoprecipitation}

A RNA immunoprecipitation was used to analyze whether XIST was associated with the RNA-induced silencing complex (RISC). A549 and H1299 cells were lysed and incubated with RIPA buffer containing magnetic beads conjugated with human anti-Argonaute2 (Ago2) antibody (Millipore). Normal mouse IgG (Millipore) was used as a negative control. Samples were incubated with Proteinase K, and then immunoprecipitated RNA was extracted. Purified RNA was subjected to qRT-PCR analysis.

\section{Xenograft mouse model.}

LV-NC- or LV-shXIST-infected A549 cells $\left(2 \times 10^{6}\right)$, were subcutaneously injected into nude mice. Tumor volumes were measured $\left(0.5 \times\right.$ length $\times$ width $\left.^{2}\right)$ in mice every three days. After 4 weeks, the mice were sacrificed, and the tumors were removed and weighed. All experiments were performed and conformed to the Principles of Laboratory Animal Care (National Society for Medical Research). 
Statistical analysis

The data are presented as mean \pm standard error from at least three separate experiments. Differences among groups were analyzed by Student's $t$-test or one-way ANOVA. P values $<0.05$ were considered statistically significant. All statistical analyses were conducted using SPSS 17.0 (SPSS Inc., Chicago, IL, USA).

\section{Results}

XIST expression is upregulated in NSCLC tissues and cell lines

To determine the role of XIST in NSCLC progression, we first measured XIST expression levels in four human NSCLC cell lines (A549, H1299, Calu-3 and SK-MES-1) and a human bronchial epithelial cell line (BEAS-2B). Higher expression of XIST was observed in all NSCLC cell lines tested compared with the human bronchial epithelial cell line (Fig. 1A). Next, we detected XIST expression in 30 pairs of NSCLC and adjacent normal lung tissues. As shown in Fig. 1B, XIST expression was higher in NSCLC tissues than in paired adjacent normal lung tissues. All data indicated that XIST was frequently up-regulated in NSCLC.

\section{XIST knockdown inhibited NSCLC malignancy}

To explore whether XIST has a role in the pathogenesis of NSCLC, XIST was silenced in the high-expressing A549 and H1299 cell lines using siXIST; siNC was the negative control (Fig. 2A). We found that the expression of proliferation- and invasion-related proteins, including proliferating cell nuclear antigen (PCNA), recombinant cyclin D1 (CCND1), B-cell lymphoma-2 (Bcl2), and matrix metallopeptidase 9 (MMP9) was decreased after XIST knockdown (Fig. 2B). As demonstrated by MTT assays, repression of XIST decreased the cell viability in A549 and $\mathrm{H} 1299$ cells (Fig. 2C). Flow cytometry indicated significant induction of S arrest and promotion of G0/G1 fraction in A549 and H1299 cells transfected with siXIST (Fig. 2D). Similarly, XIST knockdown also induced significantly higher levels of apoptosis (Fig. 2E) and suppressed cell migration (Fig. 2F) and invasion (Fig. 2G) in A549 and H1299 cells compared with the negative control group. These results revealed that XIST knockdown inhibited several malignancy parameters of NSCLC cells in vitro.

\section{XIST knockdown suppressed NSCLC tumorigenesis in vivo}

We established a mouse xenograft model to examine whether XIST knockdown could impact tumor growth in vivo. A549 cells infected with LV-shXIST or LV-NC were subcutaneously injected into nude mice. After 30 days, the tumor size was decreased in the LV-shXIST group compared with the control group (Fig. 3A). Knockdown of XIST suppressed

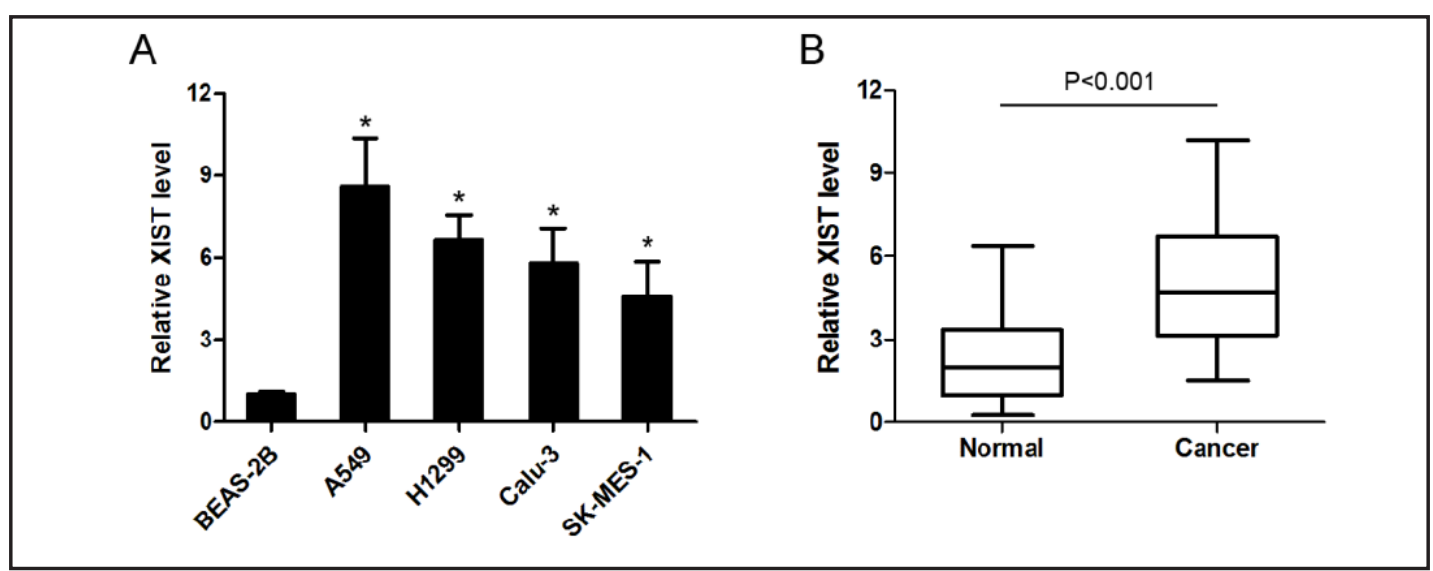

Fig. 1. XIST is upregulated in NSCLC cell lines and tissues. (A) XIST expression was detected in NSCLC cell lines and one human bronchial epithelial cell line (BEAS-2B). (B) XIST expression was measured in NSCLC tissues and normal tissues by qRT-PCR analysis. 
A

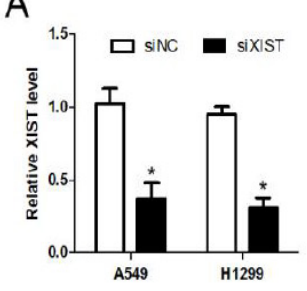

C

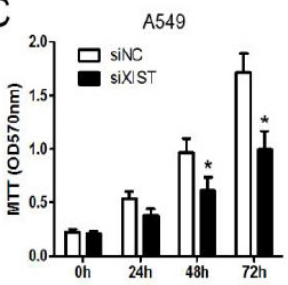

D

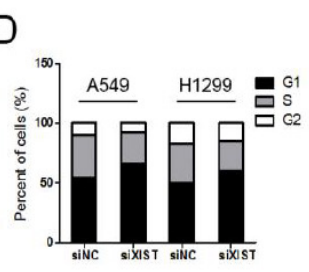

B
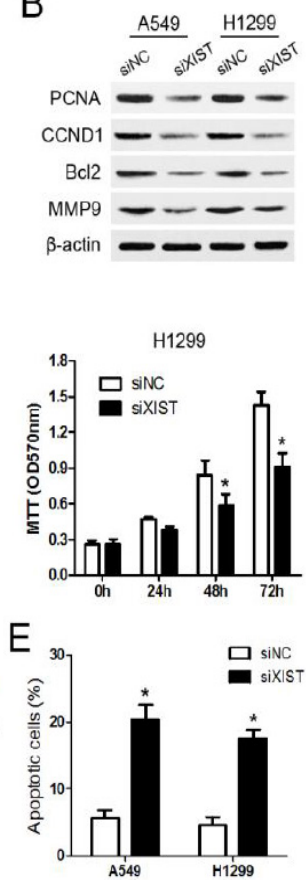

$\mathrm{F}$
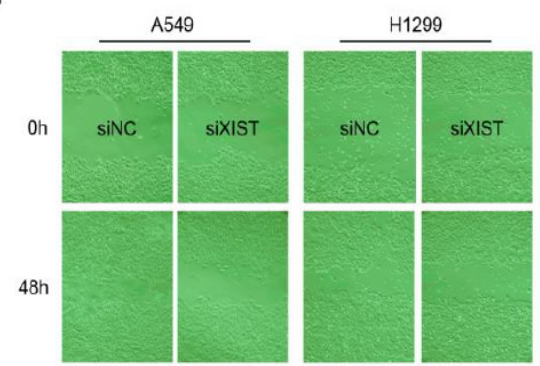

G
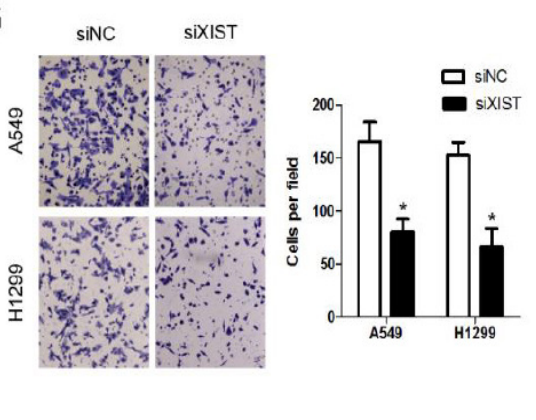

Fig. 2. XIST knockdown inhibited NSCLC cell proliferation and invasion. (A) XIST knockdown in A549 and H1299 cells was confirmed by qRT-PCR. (B) The expression of cell proliferation- and invasion-related proteins was measured by western blot in A549 and H1299 cells transfected with siXIST or siNC. (C) MTT assays were used to measure cell viability. (D and E) Cell cycle and apoptosis were analyzed in NSCLC cells transfected with siXIST or siNC. (F and G) Migration and invasion in NSCLC cells were detected using wound healing and transwell invasion assays, respectively.

Fig. 3. XIST knockdown suppressed tumor growth in vivo. (A) Photographs of tumors. (B) Tumor volumes and (C) tumor weights from xenografts with A549 cells infected with LV-shXIST or LV-NC. (D) Ki67 immunohistochemistry.

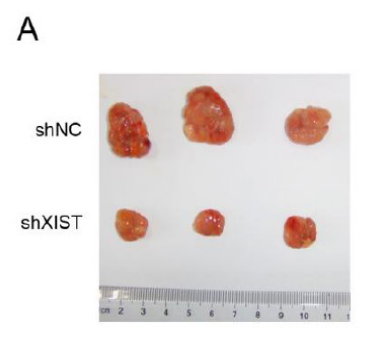

B
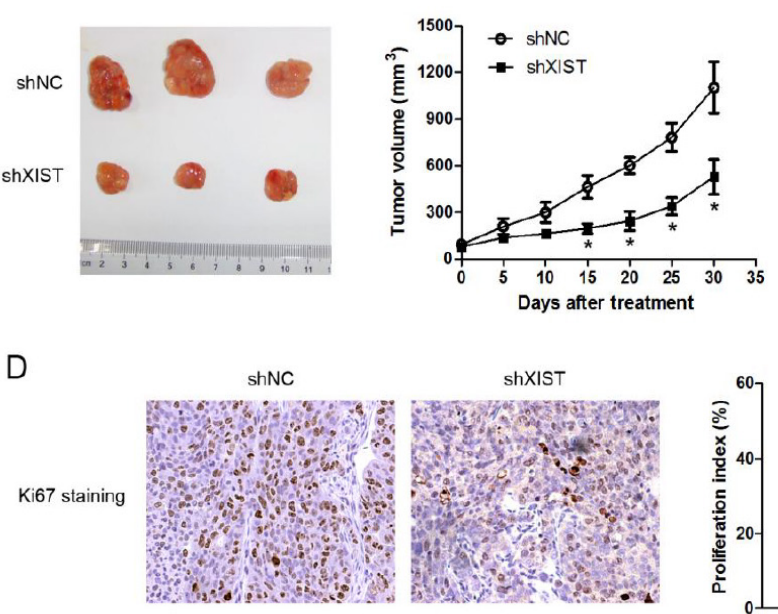

C

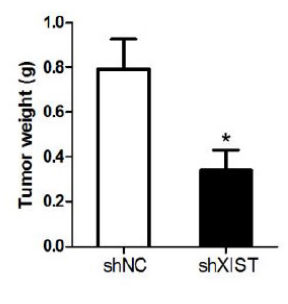

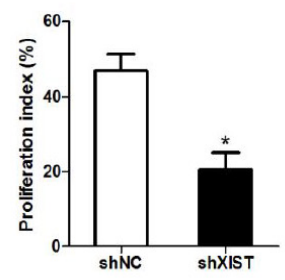

tumor growth compared with the negative control (Fig. 3B). Similar results were observed for tumor weight (Fig. 3C). Moreover, tumor sections from the LV-shXIST group exhibited weaker Ki67 staining, a marker of cell proliferation, than those from the control group, indicating that XIST knockdown inhibited NSCLC growth (Fig. 3D). These results showed that knockdown of XIST expression could suppress tumor growth in vivo. 
A

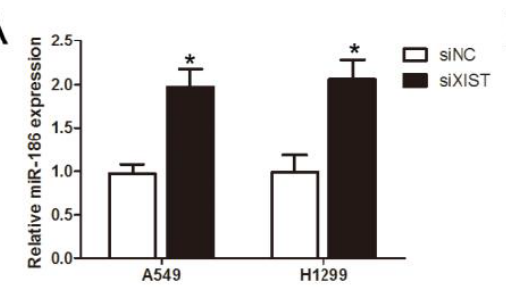

C

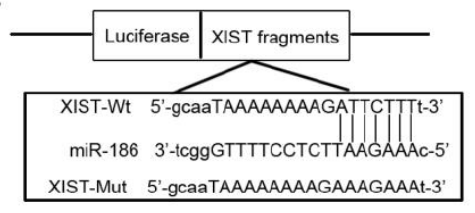

D

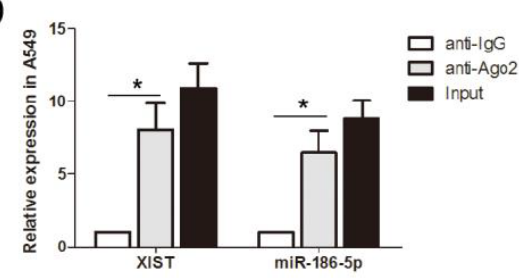

B
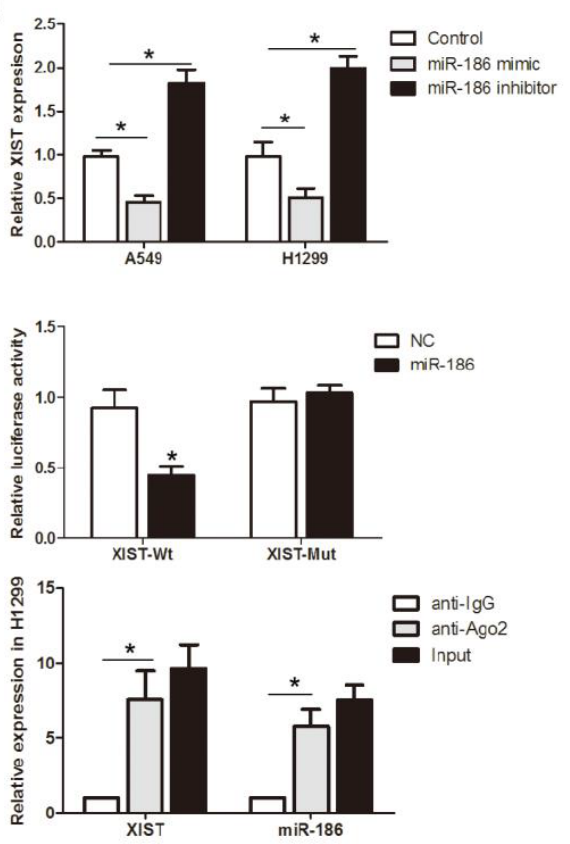

Fig. 4. Reciprocal repression between XIST and miR-186-5p. (A) Effects of siXIST on the miR-186-5p expression in A549 and H1299 cells. (B) Effects of miR-186-5p mimics or inhibitors on XIST expression in A549 and H1299 cells. (C) Schematic representation of the predicted binding sites for XIST, and the mutagenesis design for the reporter assays. (D) Relative miR-186-5p and XIST expression, presented as fold enrichment in Ago2 relative to normal IgG immunoprecipitates.

\section{Reciprocal repression between XIST and miR-186-5p}

Recently, a competitive RNA (ceRNA) hypothesis has been proposed and several studies have suggested that there are interactions between IncRNAs and miRNAs in cancer [17-19]. It has been found that miR-186-5p functions as a tumor suppressor in human NSCLC [20]. To explore whether XIST affects NSCLC proliferation and invasion by regulating miR-186-5p expression, we measured miR-186-5p expression after silencing the expression of XIST. We found that the expression of miR-186-5p was higher in the siXIST group than in the control group (Fig. 4A). Next, we detected XIST expression after overexpression and inhibition of miR-186-5p to confirm whether XIST was also negatively regulated by miR-186-5p, the XIST expression was decreased in miR-186-5p overexpression group, while enhanced inmiR186-5p inhibition group (Fig. 4B). These results revealed that XIST and miR-186-5p have a reciprocally repressive relationship. To further confirm the relationship between XIST and miR-186-5p, we performed the following experiments. Firstly, we used the bioinformatics software predicted that XIST harbor one miR-186-5p binding site. To verify that XIST was a target of miR-186-5p, the wild-type and mutant XIST cDNA sequence containing the putative miR-186-5p recognition site was cloned and co-transfected into cells with miR-186-5p mimics or negative control. Luciferase activity assays showed that miR-186-5p suppressed the activity of the wild-type but not that of the mutant XIST (Fig. 4C). These results suggest that interaction between XIST and miR-186-5p has reciprocal effects.

It is well defined that miRNA plays its function by binding to Ago2, a crucial component of the RNA-induced silencing complex (RISC) complex necessary for miRNA-mediated gene silencing, and potential miRNA targets can be isolated from this complex after Ago2 co-immunoprecipitation [21, 22]. To detect if XIST and miR-186-5p are in the same RISC complex, we performed an RNA-binding protein immunoprecipitation (RIP) assay to pull down endogenous Ago2-containing miRNP complexes and their associated miRNA in NSCLC cells. XIST and miR-186-5p were enriched in Ago2 immunoprecipitates compared with 
Fig. 5. miR-186-5p overexpression blocked the oncogenic functions of XIST in NSCLC. A549 cells were transfected with miR-1865p, XIST, miR-186-5p + XIST and negative control, respectively. (AD) Cell proliferation (at $72 \mathrm{~h}$ ), cell cycle, apoptosis and invasion were measured using MTT, flow cytometry, annexin $\mathrm{V}$, and the transwell assay, respectively.

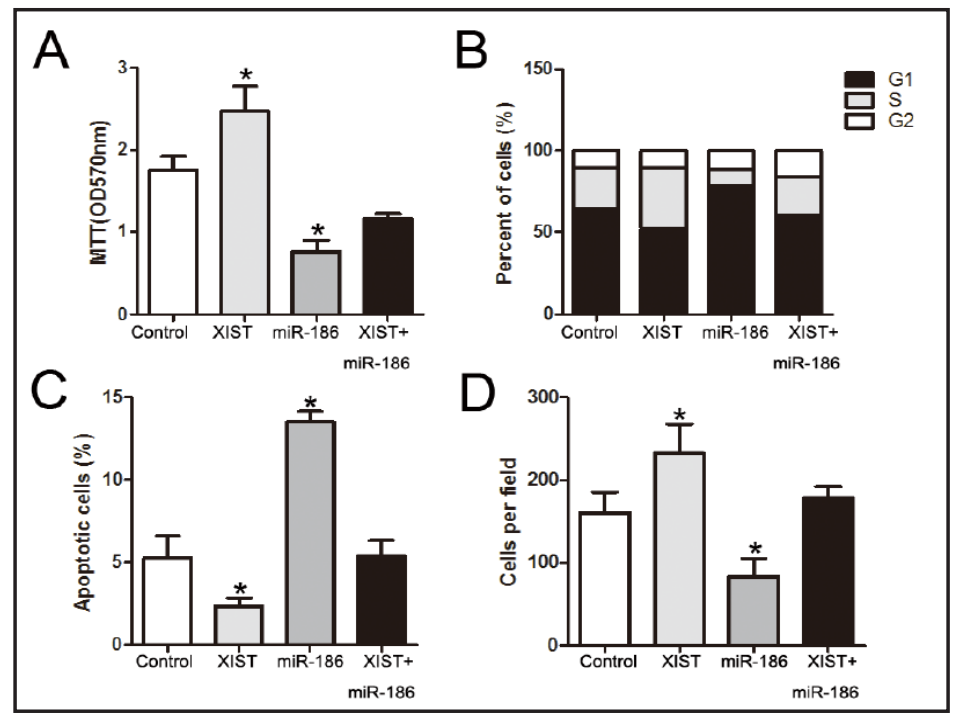

control IgG immunoprecipitates (Fig. 4D). These findings demonstrated that XIST and miR186-5p are probably in the same RISC complex in NSCLC cells.

miR-186-5p mediates the tumor-suppressive effects of XIST knockdown on NSCLC proliferation and invasion

To analyze the importance of miR-186-5p in XIST-mediated NSCLC proliferation and invasion, we overexpressed miR-186-5p in A549 cells transfected with XIST. The results showed that miR-186-5p overexpression blocked the oncogenic effects of XIST on cell proliferation and invasion (Fig. 5A-5D), suggesting that the effects of XIST on NSCLC growth and invasion are partially mediated by miR-186-5p.

\section{Discussion}

Numerous studies have shown that ncRNAs play key roles in cancer pathogenesis and could offer a new insight into the biology of the disease. ncRNAs are divided into two groups, small non-coding RNAs and IncRNAs. Over the past decade, small non-coding RNAs, such as miRNAs, have been extensively studied, and their roles in gene regulation and cell function have been elucidated in numerous cancers [23-27]. Recent studies have indicated that lncRNA also participate in several different biologic processes and play oncogenic or tumor suppressive roles during tumorigenesis [28-30]. However, the roles of IncRNAs in NSCLC remain largely unknown. Understanding the molecular mechanism through which lncRNAs function would accelerate the development of IncRNA-directed diagnostics and therapeutics against cancers [31].

Previous study has been reported that the IncRNA XIST was upregulated in NSCLC [16]. However, the role and mechanism of XIST in NSCLC remained unknown. In this study, we confirmed that XIST expression was upregulated in NSCLC cell lines and tissues. Furthermore, we determined the effects of XIST knockdown on the biological behaviors of NSCLC cells in vitro, indicating that XIST knockdown suppressed NSCLC cell proliferation and invasion. We also confirmed that XIST knockdown inhibited tumor growth in vivo. All data showed that XIST knockdown exerted suppressive effects in NSCLC.

Recently, ceRNA hypothesis has been proposed and several studies have confirmed the interaction between IncRNA and miRNA in cancer. An example of this type of regulation was exemplified by cancer susceptibility candidate 2 (CASC2), whose decreased expression is in part due to its oncogenic effects on the expression and activity of miR-18a in colorectal cancer [32]. Similar studies have revealed that long noncoding RNA activated by TGF- $\beta$ (lncRNAATB) upregulates ZEB1 and ZEB2 expression by competitively binding the miR-200 family, 
which induces epithelial-mesenchymal transition (EMT) and invasion in hepatocellular carcinoma [33]. We hypothesized that XIST may act as a ceRNA in NSCLC. Bioinformatics databases were used to search for miRNAs that contained XIST binding sites. miR-186-5p was confirmed as one of the miRNAs which could regulate XIST expression in NSCLC. We found that there was reciprocal repression between XIST and miR-186-5p. Moreover, we have validated the direct binding ability of the predicted miR-186-5p binding site on the XIST by luciferase reporter assays. We also indicated that XIST and miR-186-5p are in the same RISC complex. We further found that miR-186-5p could rescue the effects that knockdown of XIST exerted. These results revealed that XIST knockdown may suppress NSCLC proliferation and invasion through acting as miR-186-5p sponge and indicated a novel XIST-miR-186-5p regulatory axis in NSCLC.

Taken together, our data show that XIST expression is increased in NSCLC cell lines and tissues. XIST knockdown inhibited cell proliferation and invasion as well as inducing apoptosis in NSCLC. Moreover, miR-186-5p mediated the tumor-suppressive effects that knockdown of XIST exerted. Our study suggests a novel regulatory network for NSCLC proliferation, invasion and provides new information for use in the diagnosis and treatment of NSCLC.

\section{Disclosure Statement}

None.

\section{References}

Siegel R, Ma J, Zou Z, Jemal A: Cancer statistics, 2014. CA Cancer J Clin 2014;64:9-29.

Siegel RL, Miller KD, Jemal A: Cancer statistics, 2015. CA Cancer J Clin 2015;65:5-29.

Djebali S, Davis CA, Merkel A, Dobin A, Lassmann T, Mortazavi A, Tanzer A, Lagarde J, Lin W, Schlesinger F, Xue C, Marinov GK, Khatun J, Williams BA, Zaleski C, Rozowsky J, Roder M, Kokocinski F, Abdelhamid RF, Alioto T, Antoshechkin I, Baer MT, Bar NS, Batut P, Bell K, Bell I, Chakrabortty S, Chen X, Chrast J, Curado J, Derrien T, Drenkow J, Dumais E, Dumais J, Duttagupta R, Falconnet E, Fastuca M, Fejes-Toth K, Ferreira P, Foissac S, Fullwood MJ, Gao H, Gonzalez D, Gordon A, Gunawardena H, Howald C, Jha S, Johnson R, Kapranov P, King B, Kingswood C, Luo OJ, Park E, Persaud K, Preall JB, Ribeca P, Risk B, Robyr D, Sammeth M, Schaffer L, See LH, Shahab A, Skancke J, Suzuki AM, Takahashi H, Tilgner H, Trout D, Walters N, Wang H, Wrobel J, Yu Y, Ruan X, Hayashizaki Y, Harrow J, Gerstein M, Hubbard T, Reymond A, Antonarakis SE, Hannon G, Giddings MC, Ruan Y, Wold B, Carninci P, Guigo R, Gingeras TR: Landscape of transcription in human cells. Nature 2012;489:101-108.

- 4 Nitu R, Rogobete AF, Gundogdu F, Tanasescu S, Boruga O, Sas A, Popovici SE, Hutanu D, Pilut C, Sarau CA, Candea AC, Stan AT, Moise LM: microRNAs Expression as Novel Genetic Biomarker for Early Prediction and Continuous Monitoring in Pulmonary Cancer. Biochem Genet 2017 Jan 9.

5 Tsai MC, Spitale RC, Chang HY: Long intergenic noncoding rnas: new links in cancer progression. Cancer Res 2011;71:3-7.

6 Tang Q Ni Z, Cheng Z, Xu J, Yu H, Yin P. Three circulating long non-coding RNAs act as biomarkers for predicting NSCLC. Cell Physiol Biochem. 2015;37(3):1002-9.

7 Hirata H, Hinoda Y, Shahryari V, Deng G, Nakajima K, Tabatabai ZL, Ishii N, Dahiya R: Long noncoding rna malat1 promotes aggressive renal cell carcinoma through ezh2 and interacts with mir-205. Cancer Res 2015;75:1322-1331.

8 Ponting CP, Oliver PL, Reik W: Evolution and functions of long noncoding rnas. Cell 2009;136:629-641.

9 He Y, Meng XM, Huang C, Wu BM, Zhang L, Lv XW, Li J: Long noncoding rnas: novel insights into hepatocelluar carcinoma. Cancer Lett 2014;344:20-27.

10 Wan L, Sun M, Liu GJ, Wei CC, Zhang EB, Kong R, Xu TP, Huang MD, Wang ZX: Long noncoding rna pvt1 promotes non-small cell lung cancer cell proliferation through epigenetically regulating lats2 expression. Mol Cancer Ther 2016;15:1082-1094.

11 Nie W, Ge HJ, Yang XQ, Sun X, Huang H, Tao X, Chen WS, Li B: Lncrna-uca1 exerts oncogenic functions in non-small cell lung cancer by targeting mir-193a-3p. Cancer Lett 2016;371:99-106. 


\section{Cellular Physiology Cell Physiol Biochem 2017;41:2221-2229 \begin{tabular}{l|l|l} 
and Biochemistry & $\begin{array}{l}\text { DOI: 10.1159/000475637 } \\
\text { Published onlIne: April 24, } 2017\end{array}$ & $\begin{array}{l}\text { C) } 2017 \text { The Author(s). Published by S. Karger AG, Basel } \\
\text { www.karger.com/cpb }\end{array}$
\end{tabular}}

12 Brown CJ, Ballabio A, Rupert JL, Lafreniere RG, Grompe M, Tonlorenzi R, Willard HF: A gene from the region of the human $\mathrm{x}$ inactivation centre is expressed exclusively from the inactive $\mathrm{x}$ chromosome. Nature 1991;349:38-44.

13 Huang YS, Chang CC, Lee SS, Jou YS, Shih HM: Xist reduction in breast cancer upregulates akt phosphorylation via hdac3-mediated repression of phlpp1 expression. Oncotarget 2016;7:43256-43266.

14 Yao Y, Ma J, Xue Y, Wang P, Li Z, Liu J, Chen L, Xi Z, Teng H, Wang Z, Li Z, Liu Y: Knockdown of long noncoding rna xist exerts tumor-suppressive functions in human glioblastoma stem cells by up-regulating mir-152. Cancer Lett 2015;359:75-86.

15 Huang KC, Rao PH, Lau CC, Heard E, Ng SK, Brown C, Mok SC, Berkowitz RS, Ng SW: Relationship of xist expression and responses of ovarian cancer to chemotherapy. Mol Cancer Ther 2002;1:769-776.

-16 Tantai J, Hu D, Yang Y, Geng J: Combined identification of long non-coding rna xist and hif1a-as1 in serum as an effective screening for non-small cell lung cancer. Int J Clin Exp Pathol 2015;8:7887-7895.

17 Liu Q Huang J, Zhou N, Zhang Z, Zhang A, Lu Z, Wu F, Mo YY: Lncrna loc285194 is a p53-regulated tumor suppressor. Nucleic Acids Res 2013;41:4976-4987.

18 Zhang Z, Zhu Z, Watabe K, Zhang X, Bai C, Xu M, Wu F, Mo YY: Negative regulation of lncrna gas5 by mir-21. Cell Death Differ 2013;20:1558-1568.

19 Juan L, Wang G, Radovich M, Schneider BP, Clare SE, Wang Y, Liu Y: Potential roles of micrornas in regulating long intergenic noncoding rnas. BMC Med Genomics 2013;6 Suppl 1:S7.

20 Li H, Yin C, Zhang B, Sun Y, Shi L, Liu N, Liang S, Lu S, Liu Y, Zhang J, Li F, Li W, Liu F, Sun L, Qi Y: Pttg1 promotes migration and invasion of human non-small cell lung cancer cells and is modulated by mir-186. Carcinogenesis 2013;34:2145-2155.

21 Karginov FV, Conaco C, Xuan Z, Schmidt BH, Parker JS, Mandel G, Hannon GJ: A biochemical approach to identifying microrna targets. Proc Natl Acad Sci U S A 2007;104:19291-19296.

-22 Gregory RI, Chendrimada TP, Cooch N, Shiekhattar R: Human risc couples microrna biogenesis and posttranscriptional gene silencing. Cell 2005;123:631-640.

23 Wu H, Liu Y, Shu XO, Cai Q: Mir-374a suppresses lung adenocarcinoma cell proliferation and invasion by targeting tgfa gene expression. Carcinogenesis 2016;37:567-575.

-24 Xu YF, Mao YP, Li YQ Ren XY, He QM, Tang XR, Sun Y, Liu N, Ma J: Microrna-93 promotes cell growth and invasion in nasopharyngeal carcinoma by targeting disabled homolog-2. Cancer Lett 2015;363:146-155.

25 Yan GJ, Yu F, Wang B, Zhou HJ, Ge QY, Su J, Hu YL, Sun HX, Ding LJ: Microrna mir-302 inhibits the tumorigenicity of endometrial cancer cells by suppression of cyclin d1 and cdk1. Cancer Lett 2014;345:3947.

26 Chen G, Shen ZL, Wang L, Lv CY, Huang XE, Zhou RP: Hsa-mir-181a-5p expression and effects on cell proliferation in gastric cancer. Asian Pac J Cancer Prev 2013;14:3871-3875.

27 Xu N, Zhang L, Meisgen F, Harada M, Heilborn J, Homey B, Grander D, Stahle M, Sonkoly E, Pivarcsi A: Microrna-125b down-regulates matrix metallopeptidase 13 and inhibits cutaneous squamous cell carcinoma cell proliferation, migration, and invasion. J Biol Chem 2012;287:29899-29908.

28 Shao Y, Ye M, Li Q, Sun W, Ye G, Zhang X, Yang Y, Xiao B, Guo J: Lncrna-rmrp promotes carcinogenesis by acting as a mir-206 sponge and is used as a novel biomarker for gastric cancer. Oncotarget 2016;7:3781237824.

-29 Zheng J, Huang X, Tan W, Yu D, Du Z, Chang J, Wei L, Han Y, Wang C, Che X, Zhou Y, Miao X, Jiang G, Yu X, Yang X, Cao G, Zuo C, Li Z, Wang C, Cheung ST, Jia Y, Zheng X, Shen H, Wu C, Lin D: Pancreatic cancer risk variant in linc 00673 creates a mir-1231 binding site and interferes with ptpn 11 degradation. Nat Genet 2016;48:747-757.

-30 Yang ZT, Li Z, Wang XG, Tan T, Yi F, Zhu H, Zhao JP, Zhou XF. Overexpression of Long Non-Coding RNA ZXF2 Promotes Lung Adenocarcinoma Progression Through c-Myc Pathway. Cell Physiol Biochem 2015;35:23602370.

31 Ma MZ, Chu BF, Zhang Y, Weng MZ, Qin YY, Gong W, Quan ZW: Long non-coding rna ccat1 promotes gallbladder cancer development via negative modulation of mirna-218-5p. Cell Death Dis 2015;6:e1583.

-32 Huang G, Wu X, Li S, Xu X, Zhu H, Chen X: The long noncoding rna casc2 functions as a competing endogenous rna by sponging mir-18a in colorectal cancer. Sci Rep 2016;6:26524.

-33 Yuan JH, Yang F, Wang F, Ma JZ, Guo YJ, Tao QF, Liu F, Pan W, Wang TT, Zhou CC, Wang SB, Wang YZ, Yang Y, Yang N, Zhou WP, Yang GS, Sun SH: A long noncoding rna activated by tgf-beta promotes the invasionmetastasis cascade in hepatocellular carcinoma. Cancer Cell 2014;25:666-681. 